\title{
The development of InterNetwork channel Emulation platform for Surgical Robot Telemanipulation control system (INSeRT)
}

\author{
Maciej Rostański, \\ Paweł Buchwald, \\ Krystian Mączka \\ University of Dąbrowa \\ Górnicza, Poland
}

\author{
Paweł Kostka \\ Silesian University of Technology, \\ Biomedical Engineering Faculty, \\ Zabrze, Poland
}

\author{
Zbigniew Nawrat \\ Foundation for Development \\ of Cardiac Surgery \\ in Zabrze, Poland
}

\begin{abstract}
In this paper, we describe the RobinHeart surgery robot development related project in which entire robot operation is supposedly done remotely, using wide area network connection. In such environment, any vision and telemanipulation data packets are subject to delay, limitations, issues and failures, as any network connections do. It has become necessary to create an internetwork emulation for the purposes of robot-related trials. The INSeRT platform has been developed to fulfill that role. INSeRT platform design is presented and discussed and first results of validation with real-life example are shown. Network channel manipulation techniques can be implemented at data-link OSI layer level, or it may be induced at network layer level - this approach relies on network layer packet manipulation and poses a promising start, as well as raises questions about whether implementing different distributions for delay or reordering is going to have significant impact on channel emulation and traffic parameters.
\end{abstract}

Index Terms-Network performance, simulation, emulator, WAN, internetwork, telemanipulation, remote operation

\section{Introduction}

$\mathrm{T}$ HE research and development presented in this paper is driven by the development of a surgical robot for long distance operation. The family of Robin Heart [1] tele-manipulators was founded in the Foundation for Development of Cardiac Surgery of Prof. Zbigniew Religa (FRK) in Zabrze, in collaboration with specialists from several academic centres (Lodz, Gliwice and Warsaw Technical Universities). According to project preliminary assumptions, the robot would have a segment-like structure allowing different configurations set up for various types of soft-tissue surgery. In particular, it should have an independent arm of the endoscopic video track with a wide range of application. The project has evolved together with the increase of experience gathered by the construction team.

In the first phase of the Polish Robin Heart Project, three robot models: Robin Heart 0, 1 \& Robin Heart 2 (Fig.1) were created [2], differing from one another by the concept of control system and mounting. Between 2007 and 2008, the Robin Heart Vision, a robot for tracking video endoscopic channel, was constructed and tested. In 2010, after only one year of work, a new model, the Robin Heart mc2 appeared in the laboratory and in the Animal Experimental Medicine Centre for in vitro and in vivo tests. The robot fulfils the role of three operators: the first \& second surgeon as well as the assistant holding the vision channel. In the same year, after completing a 2 -year project, novel mechatronic tools, the Robin Heart Uni System were created. These innovative tools allowed to put into practice the idea of using the same surgery tools both on the robotic arm, tele-controlled by the operator, and in given cases in the other way: similar to traditional laparoscopic tools - manually but driven by means of a special handle with micro-motors mechanisms.

At the same time, effective systems of Man-Machine interface including ergonomic Surgeon (Master) environment with comfortable operating position of the Surgeon, high quality vision system and intuitive contact with Master tool of tele-manipulator (including force feedback, which is still optional, in research phase) are being developed [3].

In this paper, we describe the part of the project in which entire robot operation is supposedly done remotely, using wide area network connection. In such environment, any vision and telemanipulation data packets are subject to delay, limitations, issues and failures, as any network connections do. It has become necessary to create an emulation of internetwork for the purposes of robot-related trials. The INSeRT platform has been developed to fulfill that role.

This paper is organized as follows: the questions and problems with remote long distance operations in RobinHeart are presented, then the short review on network channel simulation state-of the-art and related issues are mentioned. INSeRT platform design is presented and discussed and first results of validation with real-life example are shown. Conclusions are drawn and the most important fields of further study are pointed out and summary is described at the end.

\section{Telemanipulation Control System for Long Distance}

Operation by Means of Robin Heart Family of Surgery Robotic Manipulators

Telemanipulator invented for less, minimally invasive cardiac surgery is a computer-controlled device, located between surgeon's hands and the tip of a surgical instrument or endoscopic vision channel. Basic requirements for this device are stable operative field of view, direct surgeon control and high level of precision. Main advantages, motivating the introduction of tele-manipulators to minimal invasive surgery field are also:

- Scaling of movement between Master console and Slave arm

- Filtering and tremor removing 

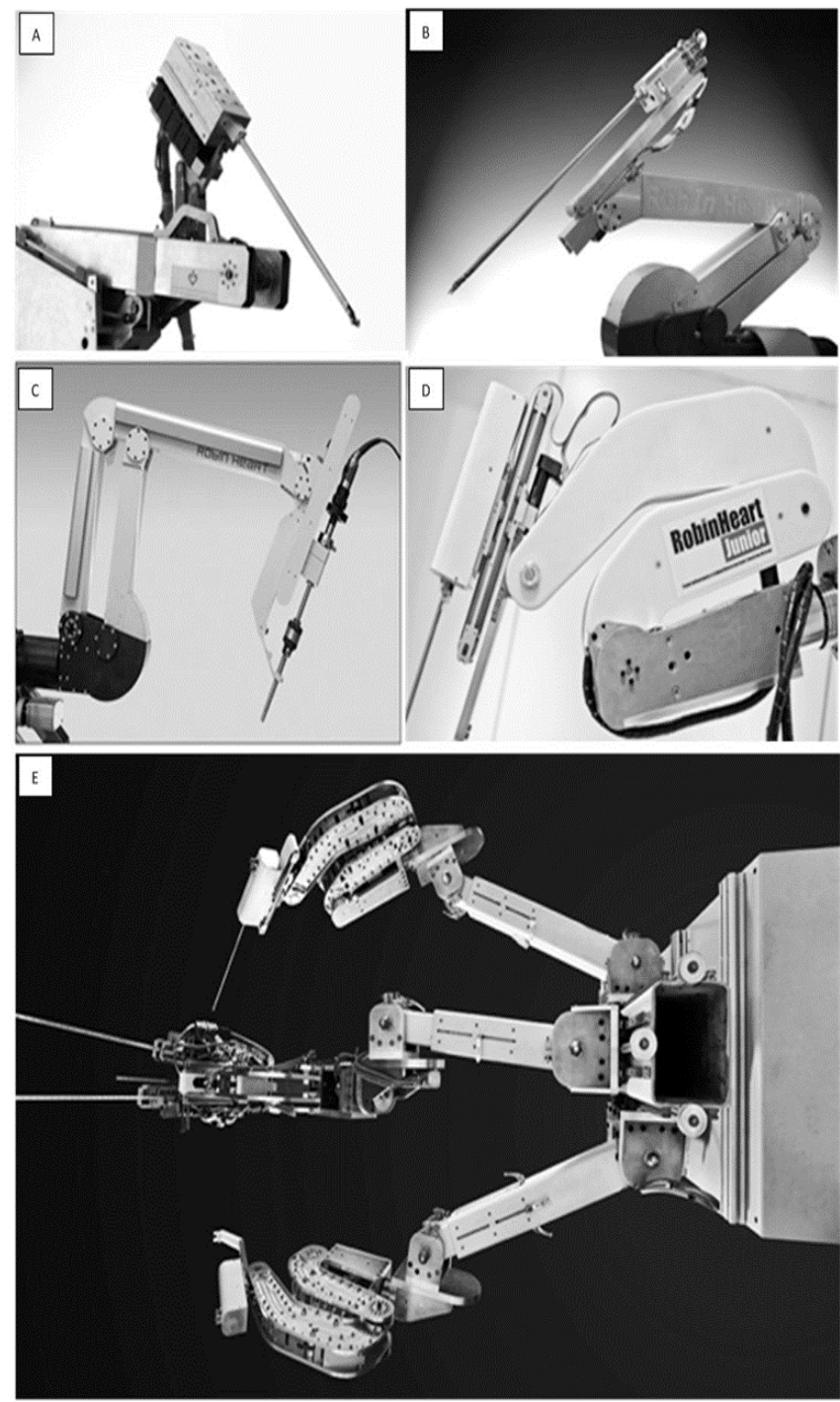

Fig 1. Progress in RobinHeart project development. - The Robin Heart family robots.: Robin Heart 0 (A), Robin Heart 1(B), Robin Heart Vision (C), Robin Heart Junior (D), Robin Heart $\mathrm{mc}^{2}(\mathrm{E})$

- Comfort of surgeon work improvement with high quality HD (stereoscopic) imaging from operation field with zooming and filtering

General structure of Master-Slave (Operator-Robot arm) teleoperation system with video and manipulation data channel is presented on Fig. 2. Optional force-feedback track is presented, that is used to transfer tool-tissue interaction to Surgeon/Operator.

In this type of robotic arm navigation the slave manipulator mimics movements of master controller, driven by Surgeon/Operator (Fig. 3). A brushless DC motors working in connection with the robust local CAN bus with dedicated low level controllers were used as a driven units for every arm with four degree of freedom. Control system exchange data between master and slave part of control system, which process and transfer data by means of network shared variables. Both video transmission as well as manipulation transmission channels performances are closely related to out-

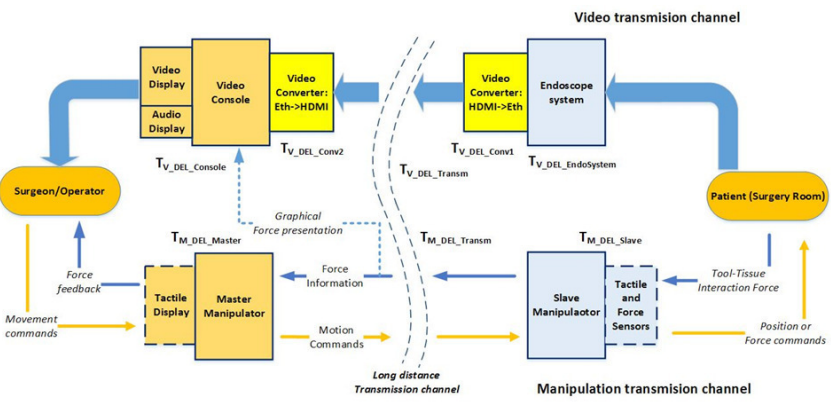

Fig 2. General structure of Operator-Robotic_Arm bidirectional data transfer: video and manipulation channel

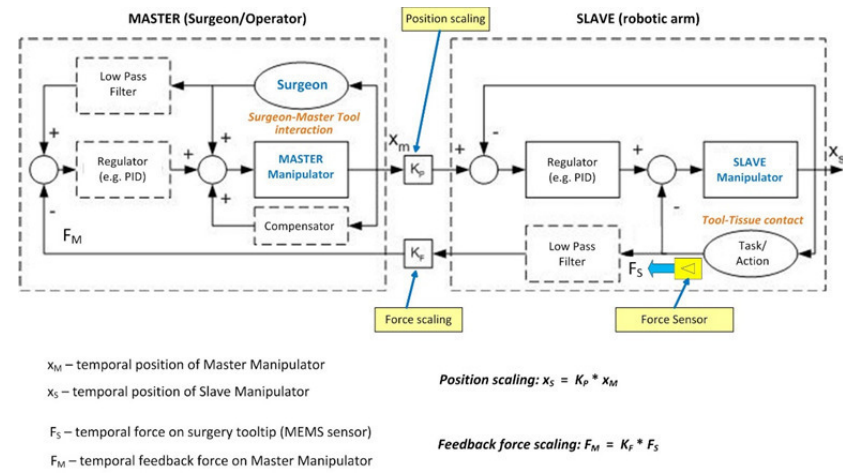

Fig 3. Bilateral, Master-Slave control system structure of Robin Heart Robot

come performance of an operator. In case of setting up any of these channels (and practically that would mean both of them) using internetwork, the network channel performance characteristic becomes critical to the system.

\section{Selected Issues of the Network Channel Emulation}

The need for analysis and simulation of network traffic is constantly increasing. A large number of studies in this field derives from the need to improve the quality of services (QoS), complete the service level agreement (SLA) or ensure proper network security level. As a first example, authors in [4] discuss the characteristics of actual Internet traffic in the context of flows and investigates the cause for the high fluctuation in the number of flows. They defined and analyze measurement metrics (such as link utilization, packet arrival rate, and the number of flows) and also test it by the use of university network with over 6000 end hosts and servers.

It is worth mentioning that types and patterns of actual network traffic flows changed dramatically. As the authors show in [5] the proportion of traditional traffic (determined by well-known port based flows) is decreasing for $\mathrm{p} 2 \mathrm{p}$ (peerto-peer) traffic or other media as streaming or network gaming therefore a better method is to classify the traffic according to application layer programs. The authors indicates that it has become difficult to detect newly developed Internet applications which use random port numbers rather than static and registered ports. 
Many aspects of traffic characterization and the dynamics and patterns of network usage was discussed by the authors in [6]. Authors proposed characterization of the traffic by linking the flow measurement architecture with the estimation algorithm. Developed framework was used to estimate the distribution and sample space of the underlying traffic by the use of nonparametric Parzen window (which is an information theory technique, possible to use for classification with various types of data sets [7]). Köandgel in [8] shows how important is characteristic of networks by the use of one-way delay measured, or taken, between any two points in the network. At his work author highlights that precision of one-way delay calculation for flow data depends on the capturing devices, especially on theirs timestamp resolution. Also, [9] presents distributions of flow length, packet size, throughput, for the popular and bandwidth consuming applications as stochastic phenomenon. Flow duration, flow bytes, packet size for popular network applications in the probability density function was presented for the flows collected at several locations on a corporate Intranet.

The classification of network traffic may be performed using machine learning approaches [10]. Network traffic classification can be used for network management (automated problems detection) or automated intrusion detection systems. The authors compare the research in this area. Very interesting subject is the collation of features, used by various researchers, to describe the Internet traffic dataset.

\section{System Design AND TeChNicAl IMPLEMENTATION}

Taking into account presented works, the most important distinguished features included:

- the necessity of control over the basic parameters of the network connection, in compliance with [11] and just like previously discussed in [12] and [13]: delay (latency), data transmission rate (throughput), lost packets ratio (packet loss) and delay variation (jitter) - this requirements form and review phases were conducted with methods described by one of authors in [14];

- as the requirements elicitation process has proven, specific system functionality has been necessary, which are pre-defined sets of network parameters and their variability trends over time (more on this subject below);

- the system should be able to gather information about the network parameters induced into channel in specific moment in time - the availability of such data will help to identify the correlation between the parameters of telemanipulation channel and assumed measure factors of remote controller operation.

Due to specific project stakeholders demands, the requirement of solution simplicity and capability of operation on available common hardware and software infrastructure has also been considered a 'must-have' (entire system had to be mobile and easily transferred, as well as we want to maintain the positive effect on system integration with other components).

The research and business enquiries allowed to distinguish two groups of solutions. Network channel manipulation tech- niques can be implemented at data-link OSI layer level, or it may be induced at network layer level, with most important examples of:

- Linux operating system kernel providing couple of built-in and patched capabilities, such as $t c$ and netem package, Iptables statistics and/or random module, Iproute 2 packet capabilities exploiting mark in iptables software;

- FreeBSD operating system's packet dummynet, providing similar capabilities;

Both concepts - bridge (Layer 2) or router (Layer 3) based, provide desired functionality in different manners, which will be the subject of another authors' study. Taken into account the requirement of easy implementation in virtualized environment and the API functionality, the layer 3 approach has been chosen. There are a couple of entire similar systems as well, such as NIST's project NISTNet (no longer maintained), or Tata's Performance Engineering Research Centre WANem (The Wide Area Network emulator) which was actively taken into consideration, but the decision was made to create own solution, with API dedicated for Robin surgical robot environment and capable of data collection for channel testing purposes. Due to Linux popularity and for stability, CentOS OS 6.6 has been recommended for implementation.

The logical network infrastructure is presented on Figure 4 .

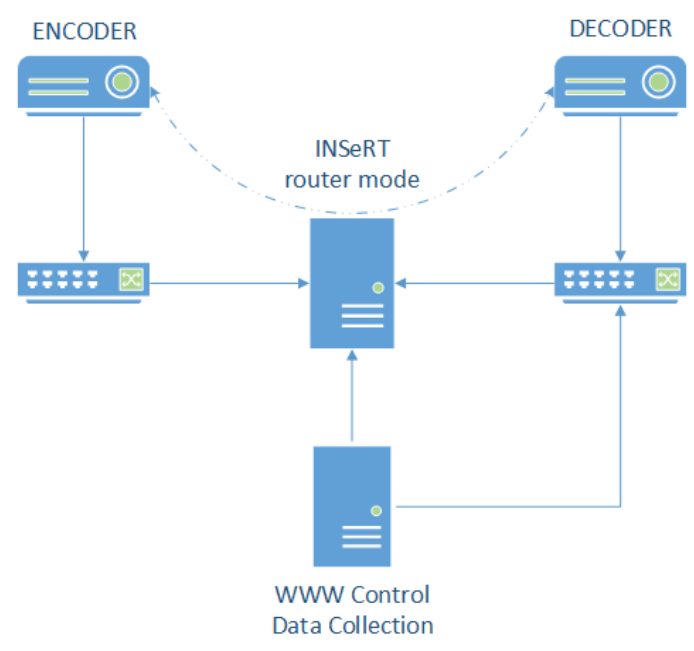

Fig 4. . INsERT system topology

Due to the need for the reduce of the interaction of the transmission line emulator with additional services such as HTTP graphical configuration module or database for connection parameters collection, they have been implemented on a separate server. Communication between a web application that offers GUI functions and module responsible for traffic manipulation takes place on a separate, dedicated channel. Commands are transferred with SSH2 protocol, which allows the use of a wide range of solutions, including certificates, so storing passwords in configuration files is avoided completely. Remote connection using SSH2 library 
enables standards console stream input and thus the introduction of any previously prepared user commands responses to an event related to changes in network parameters.

\section{Validation of Network Channel Emulation with INsERT}

Two network interfaces are used to manipulate outgoing traffic, thus enabling asymmetrical network connection emulation (by varying parameters for both directions). Example of basic manipulation is presented in Table 1 .

TABLE 1.

BASIC TESTBED SET EXAMPLE

\begin{tabular}{lll}
\hline Parameter & Values set & $\begin{array}{l}\text { Values } \\
\text { measured }\end{array}$ \\
\hline Latency & $100.0 \mathrm{~ms}$ & $104.120 \mathrm{~ms}$ \\
\hline Packet loss & $10 \%$ & $9 \%$ \\
\hline Jitter & $50 \mathrm{~ms}$ & $46.541 \mathrm{~ms}$ \\
\hline
\end{tabular}

Currently, after test trials, three basic sets, or link categories, were defined, illustrating typical internetwork connections that might be used for telemanipulation and/or vision channels:

- Good quality connection - corresponding to leased WAN line;

- Limited quality connection - corresponding to channel realized using Internet;

- Problematic connection - corresponding to 'challenged links', for example cellular or even satellite connection.

Those three categories were implemented as a presets of parameters, using simple statistical models of network traffic. Statistical characteristics are based on traffic recorded during experiments with every channel type, but those simple models do not take into the account the non-standard delay distribution, or correlation for packets delay, jitter or loss (which in turn do not emulate burst losses, for example) yet.

The example of limited quality connection emulation with real-life case study as a comparison is shown on Fig. 5 (TCP time/sequence graph) and Fig.6 (throughput). The simplicity of a model is visible on throughput graph comparison in particular - there is much more variation in real-life scenario, however the transmission characteristic is similar.

\section{Conclusions and Summary}

In presented article, the design and implementation process of internetwork channel emulation for surgical robot telemanipulation experiment was presented, as well as the discussion on various methods, issues and future possible work. The system is functional and provides means for emulating real-life scenarios when using internetwork links for remote operation of equipment using video and telemanipulation channels. System provides functionality for traffic manipulation, allowing to simulate different kinds of network links and situations. Open research question, to be pursued in further work, is whether implementing different distributions for delay, reordering, and/or packet loss is going to have significant impact on channel emulation and traffic parameters.

The INsERT system concept assumes that beside traffic characteristics manipulation, the system is designed for collecting the data on network parameters set when working remotely with surgical robot. The maintenance of ergonomic conditions of surgeon's work is essential to surgical robot system and proper assessment of the network channel conditions impact on any activity is crucial to any experiments on that matter. The determination of the minimum acceptable channel parameters is necessary to anticipate problems with the telemanipulation track, based on changing network traffic characteristics. Thanks to implemented system, it becomes
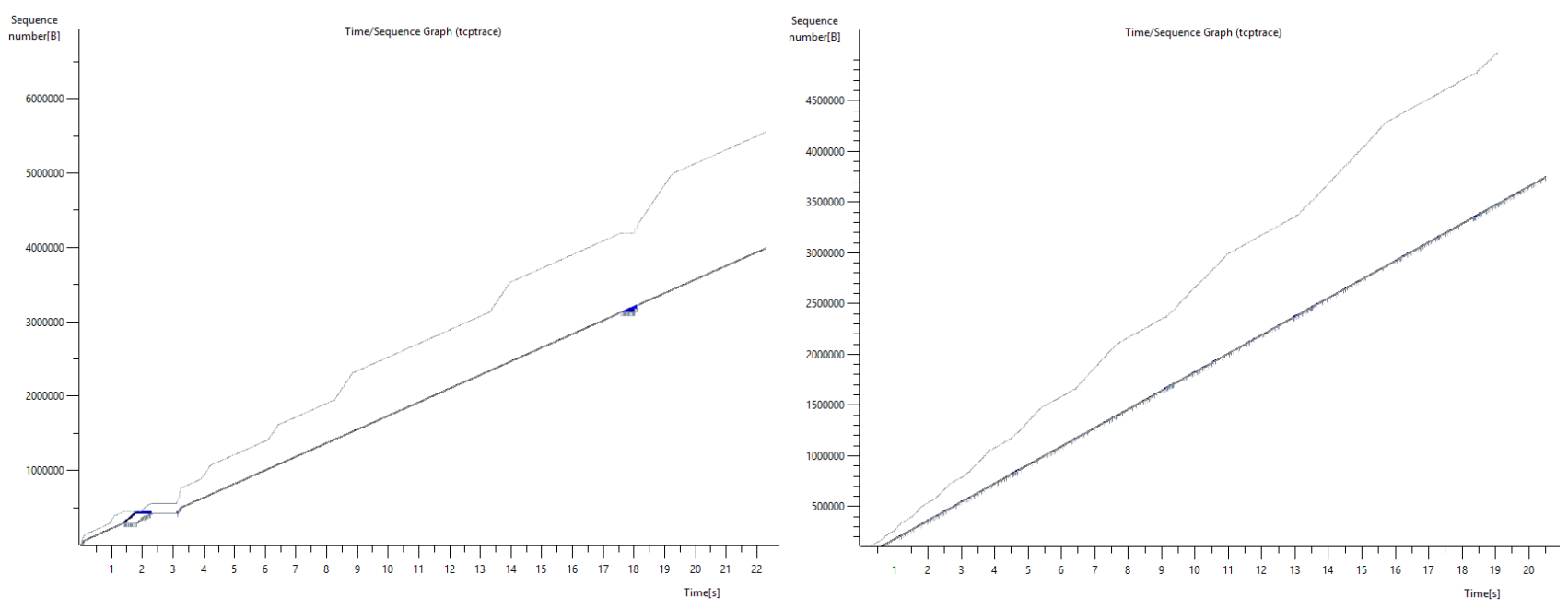

Fig 5. General structure of Operator-Robotic_Arm bidirectional data transfer: video and manipulation channel 

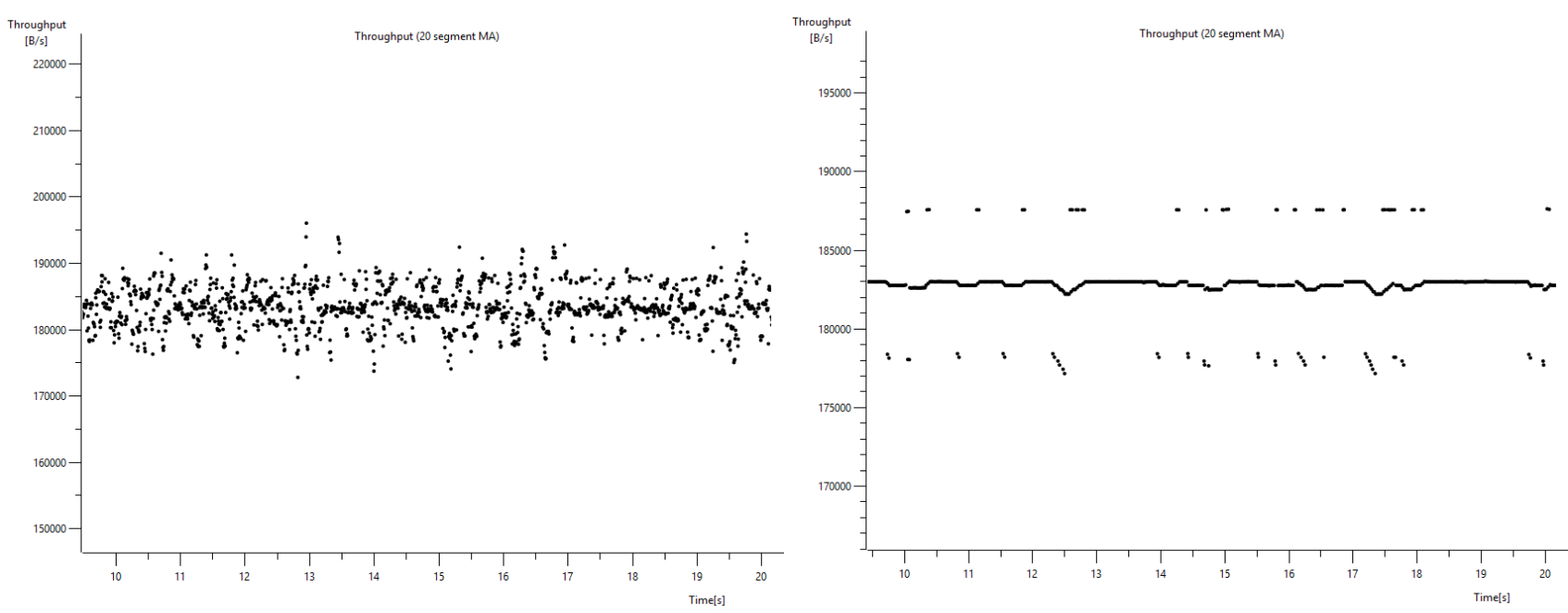

Fig 6. General structure of Operator-Robotic_Arm bidirectional data transfer: video and manipulation channel

possible to designate parameters values in an empirical manner with exercises with real surgeons in controlled training environment. Obtained data will allow for network link validation for surgical robot vision and telemanipulation purposes in real-time. Trial studies with control group of surgeon students were already conducted and will be covered in extensive manner in the future. Current research connected with the studies of influence of network parameters values on telemanipulation performance are tested on new RH Tele TM arm (Fig. 7).

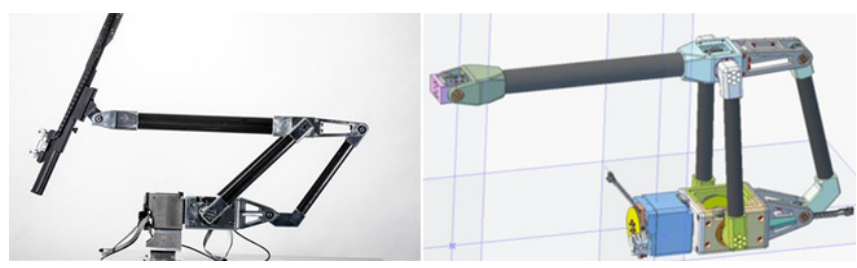

Fig 7. . RH Tele ${ }^{\mathrm{TM}}$ arm, made from carbon base, light materials (final arm and project)

\section{ACKNOWLEDGEMENTS}

This work is a part of project „,TeleRobinSurgery - Creating and study on new technical systems for remote controlled surgical procedures by means of Robin Heart family telemanipulators" (ID 181019) financially supported by Polish National Center of Reseach and Development, based on contract nb 1/A3/2/2012.

\section{REFERENCES}

[1] Nawrat, Z. and Kostka, P. (2006), Polish cardio-robot 'Robin Heart' System description and technical evaluation. Int. J. Med. Robotics Comput. Assist. Surg., 2: 36-44. doi: 10.1002/rcs.67
[2] Nawrat, Z. and Koźlak, M. (2007). Robin Heart system modelling and training in virtual reality. Journal of Automation Mobile Robotics and Intelligent Systems, 1, 62-66.

[3] Kostka, P. and Nawrat, Z. (2012). Wybrane interfejsy chirurg-maszyna w strukturze systemu wizyjnego i sterowania telemanipulatorów chirurgicznych rodziny Robin Heart. Pomiary, Automatyka, Robotyka, 16, 420-423.

[4] Kim, Myung-Sup, Young J. Won, and James W. Hong. (2006). Characteristic analysis of internet traffic from the perspective of flows. Computer Communications 29.10, 1639-1652.

[5] Saroiu S., Gummadi K.P., Dunn R.J., Gribble S.D., Levy H. M. (2002). An Analysis of Internet Content Delivery Systems, Proceedings of the 5th Symposium on Operating Systems Design and Implementation (OSDI), Boston, MA, Dec. 2002

[6] Kundu S.R., Pal S., Basu K. and Das S.K. (2009). An architectural framework for accurate characterization of network traffic. IEEE Transactions on Parallel and Distributed Systems 2009;20(1):111-23.

[7] Biesiada, J., Duch, W., Kachel, A., Maczka, K., and Palucha, S. (2005). Feature ranking methods based on information entropy with Parzen windows. In: International Conference on Research in Electrotechnology and Applied Informatics (Vol. 1, p. 1).

[8] Köandgel J. (2011). One-way delay measurement based on flow data: quantification and compensation of errors by exporter profiling. In: 2011 International conference on information networking (ICOIN), 2011. pp. 25-30.

[9] Liu D, Huebner F. (2002). Application profiling of IP traffic. In: 27th annual IEEE conference on local computer networks, 2002. Proceedings. LCN 2002: 220-229.

[10] Nguyen T., Armitage G. (2008) A survey of techniques for Internet traffic classification using machine learning. Communications Surveys Tutorials, IEEE 2008;10 4): 56-76

[11] Paxson et al.: Framework for Internet Protocol Performance Metrics, RFC 2330, RFC 7312

[12] Rostanski M., Pikiewicz P. (2010). TCP Congestion Control Algorithms Performance in $3 \mathrm{G}$ networks with moving client. In: Performance Modelling and Evaluation of Heterogeneous Networks, Proceedings of 6th Working International Conference HET-NETs 2010, ISBN: 978-83-926054-4-7, pp. 379-390

[13] Grzywak A., Pikiewicz P., Rostański M.: Sieci bezprzewodowe, Wyższa Szkoła Biznesu w Dą̧rowie Górniczej, Dąbrowa Górnicza 2010, ISBN: 978-83-88936-74-6

[14] Duda J., Rostański M., Borczyk W., Grochla K. (2015). Applying Kano model into goal/requirements elicitation for crossplatform mobile content technology. In: Conference Proceedings of Strategic Management and its Support by Information Systems 2015 (in print) 